\title{
Microstructural analysis of sheared polydisperse polyhedral grains
}

\author{
David Cantor $\odot,{ }^{1, *}$ Emilien Azéma $\odot,{ }^{2,3, \dagger}$ and Itthichai Preechawuttipong ${ }^{1, \dagger}$ \\ ${ }^{1}$ Department of Mechanical Engineering, Chiang Mai University, 239 Huay Kaew Road, Chiang Mai, Thailand \\ ${ }^{2}$ LMGC, University of Montpellier, CNRS, Montpellier, France \\ ${ }^{3}$ Laboratoire de Micromécanique et Intégrité des Structures (MIST), UM, CNRS, IRSN, France
}

(Received 29 September 2019; accepted 6 May 2020; published 1 June 2020)

\begin{abstract}
This article presents an analysis of the shear strength of numerical samples composed of polyhedra presenting a grain size dispersion. Previous numerical studies using, for instance, disks, polygons, and spheres, have consistently shown that microstructural properties linked to the fabric and force transmission allow granular media to exhibit a constant shear resistance although packing fraction can dramatically change as a broader grain-size distribution is considered. To have a complete picture of such behavior, we developed a set of numerical experiments in the frame of the discrete element method to test the shear strength of polydisperse samples composed of polyhedral grains. Although the contact networks and force transmission are quite more complex for such generalized grain shape, we can verify that the shear strength independence still holds up for 3D regular polyhedra. We make a particular focus upon the role of different contact types in the assemblies and their relative contributions to the granular connectivity and sample strength. The invariance of shear strength at the macroscopic scale results deeply linked to fine compensations at the microstructural level involving geometrical and force anisotropies of the assembly.
\end{abstract}

DOI: 10.1103/PhysRevE.101.062901

\section{INTRODUCTION}

Our understanding of the physics and mechanics of granular materials has considerably stepped forward during recent years. What we had to describe as an averaged continuum medium some decades ago can be considered today as the complex mixtures of solids, voids, and fluids that they are. This progress is indeed supported by the unceasing developments of experimental and numerical methods. And, certainly, the approach that allows one to describe with more detail the geometry of grains, their interactions, and to probe their bulk and mechanical properties is, up to now, the discrete element modeling (DEM).

The DEM has become a convenient tool to explore and discover phenomena that are hardly observable in experiments. Among these phenomena, the surprising independence of shear strength upon the grain size span is the object of this article. This phenomenon has been observed in 2D simulations using disks [1-3], employing regular and irregular polygons [4], and in 3D simulations with rigid spheres [5] by some the authors of this work. The implications of such a counterintuitive observation are vast. Numerous industrial processes, engineering, and scientific disciplines deal with size varying granular materials. From geologists analyzing the mechanics of fault gauges, geotechnical engineers assessing the strength of soils, or power technology applications, the understanding of the rheology and bulk properties of size disperse materials is essential. However, along with grain size, the strength

\footnotetext{
*david.cantor@cmu.ac.th

†emilien.azema@umontpellier.fr

†tthichai.p@cmu.ac.th
}

properties of the granular media are strongly linked to the shape of grains. It has been shown in both numerical and experimental studies that grain shape has a nontrivial effect upon packing and shear properties of granular materials [6-22]. For instance, the grains presenting the more angular shapes (in opposition to rounded) do not show the highest shear strengths as one could expect. Moreover, real granular materials often present correlations between particle size and shape [23,24], making grain shape an essential element yet to be included in the analysis of the mechanical behavior of size disperse grains.

In this article, we present a systematic approach to probe the shear strength of size disperse samples composed of regular polyhedral particles. For simplicity, we fix the shape of polyhedra to octahedra. We look to generalize, or the opposite, the shear strength independence on particle size using very angular three-dimensional grains. This analysis takes into account a fine description of the contact configurations and the mechanisms of force transmission that can arise from interactions between polyhedra. Note that the transition to general three-dimensional shapes is not simple. Several issues related to the contact detection, the kinematics of 3D bodies, and the quality of the results require special consideration and a more strict numerical analysis. In effect, the numerical procedures are considerably time-consuming and require more computational and storage capacities. Throughout this paper, we point out and analyze such issues as well.

This article is organized as follows. In Sec. II, we present the numerical procedures to build and test the shear strength of samples composed of octahedra. Section III is focused on the evolution of shear strength and solid fraction up to the steady flow state. Section IV presents different parameters related to the granular connectivity and the role of distinct contact 


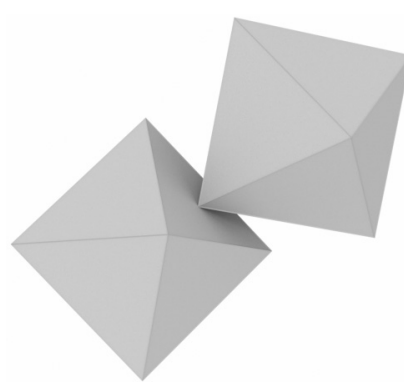

(a)

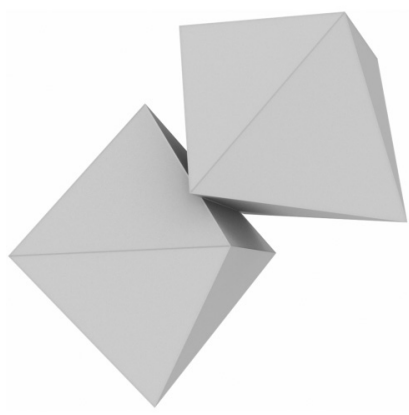

(c)

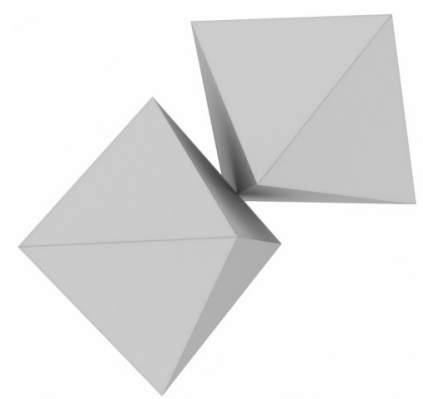

(b)

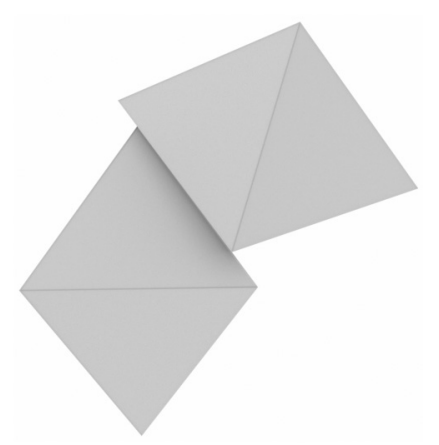

(d)
FIG. 1. Set of different contact types that appear at the interactions between convex grains. (a) vertex-face, (b) edge-edge, (c) edgeface, and (d) face-face.

between grains. In particular, we focus on the description of the contact network, its fabric properties, and anisotropies. In Sec. V, we describe the forces network and the anisotropies related to the normal and friction contact forces. Then, in Sec. VI, we present the microstructural elements behind the macroscopic strength of polyhedra. We verify at this point whether the shear resistance remains independent of the grain size span for regular polyhedra. We conclude this article with a summary and perspectives of this work.

\section{NUMERICAL MODEL}

We use the discrete element approach known as contact dynamics (CD) [25-29] to simulate assemblies of angular rigid grains. This method has systematically been used to model granular systems under a large variety of configurations, particle shapes, boundary conditions, and static and dynamic loadings [30-38]. The CD method is a nonsmooth approach based on an implicit description of the equations of motion for rigid grains obeying mutual exclusion and a dry Coulomb contact friction. The contact forces and body velocities are found, in effect, using an iterative nonlinear Gauss-Seidel algorithm.

When dealing with three-dimensional regular grains, a rich contact configuration appears given the different contact types that can occur (vertex-face, edge-edge, edge-face, face-face; see Fig. 1). Contacts vertex-vertex and vertex-edge are rare and usually discarded of the contact resolution and analysis. Note that while vertex-face and edge-edge contacts are represented by single contact points, contacts edge-face and face-face are numerically represented by two and three contact points, respectively, for an adequate resolution. Although the location of these multiple contact points may vary after a geometrical criterion, only their resultant and application point are material. Hence, the loci of the two or three contact points do not affect the dynamics of the granular system. Then, our analysis considers only one interaction between the different bodies regardless of the contact type.

Concerning the contact detection for polyhedra, we used a method based on the so-called common-plane approach proposed by Cundall [28,39]. This method iteratively looks for a plane between the touching (or potentially touching) bodies by minimizing the distances between the plane and the vertices of neighboring grains. Among different detection techniques, the common-plane method remains a robust and reasonably efficient tool when dealing with assemblies of several polyhedral grains.

We used the simulation platform LMGC90 [40,41] in which we created assemblies of 19000 regular octahedral grains set into boxes. The grains were deposited using an algorithm that places the bodies layer by layer in a semicompact packing configuration [42]. We built a set of nine samples where the grain size span varied with parameter $S$, defined as

$$
S=\frac{d_{\max }-d_{\min }}{d_{\max }+d_{\min }},
$$

from $S=0$ to $S=0.8$ in steps of 0.1 . The grain size distribution was uniform by volume fractions between the equivalent minimum and maximum equivalent diameters $\left(d_{\min }\right.$ and $d_{\max }$, respectively). Note that the equivalent diameters are also computed by particle volumes.

We initially applied an isotropic compression to the assembly up to a stable dense state in which the contact network presented just small variations in the contact forces $(<0.1 \%$ around the mean force). Note that the stable state for polyhedral grain packings has been previously analyzed by Smith et al. [43,44] and Azéma et al. [10]. Figure 2 shows two screenshots for the state of samples $S=0$ and $S=0.8$ at the end of the isotropic compression.

To apply large shear deformation, we removed the lateral walls and set periodic boundary conditions along these directions. The shear tests were performed by fixing the bottom wall and applying on the upper wall a pressure $P$ in the vertical direction and a constant velocity $v_{x}$ in the ' $x$ ' direction. The particles in contact either with the upper or the lower wall were bonded and followed the boundary constrains. No gravity was set in our experiments to avoid a differential pressure field in the samples.

These boundary conditions were set to reproduce a quasistatic shear flow. For this, we additionally used the inertial number defined as $I=\dot{\gamma} \sqrt{m / P d^{D-2}}$ with $d$ the average size of the grains, $m$ the mass of such average particle, $D$ the dimension, and the shearing rate $\dot{\gamma}=v_{x} / h_{0}$, where $h_{0}$ is the height of the sample at the beginning of the test. In all our tests, $I<1 \times 10^{-3}$ which assures a quasistatic deformation. Note that the coefficient of friction $\mu$ was set to zero during the isotropic compression but then switched to $\mu=0.4$ for the shear tests. Although our boundary conditions imply that neighboring bodies do not change very often over several time steps, the contact detection is performed on every step. 


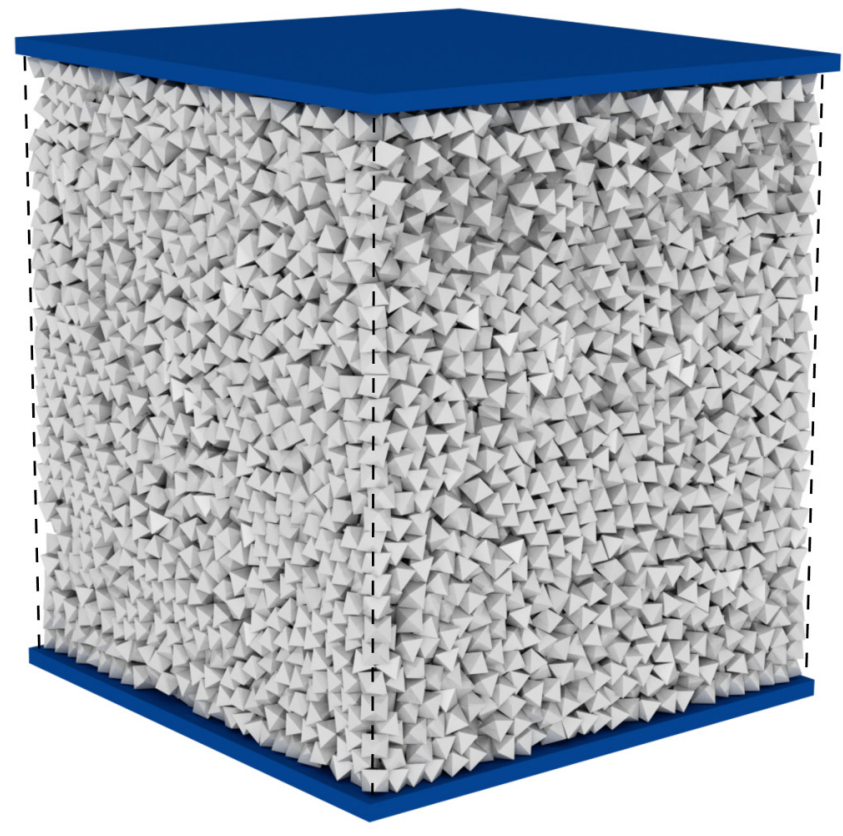

(a)

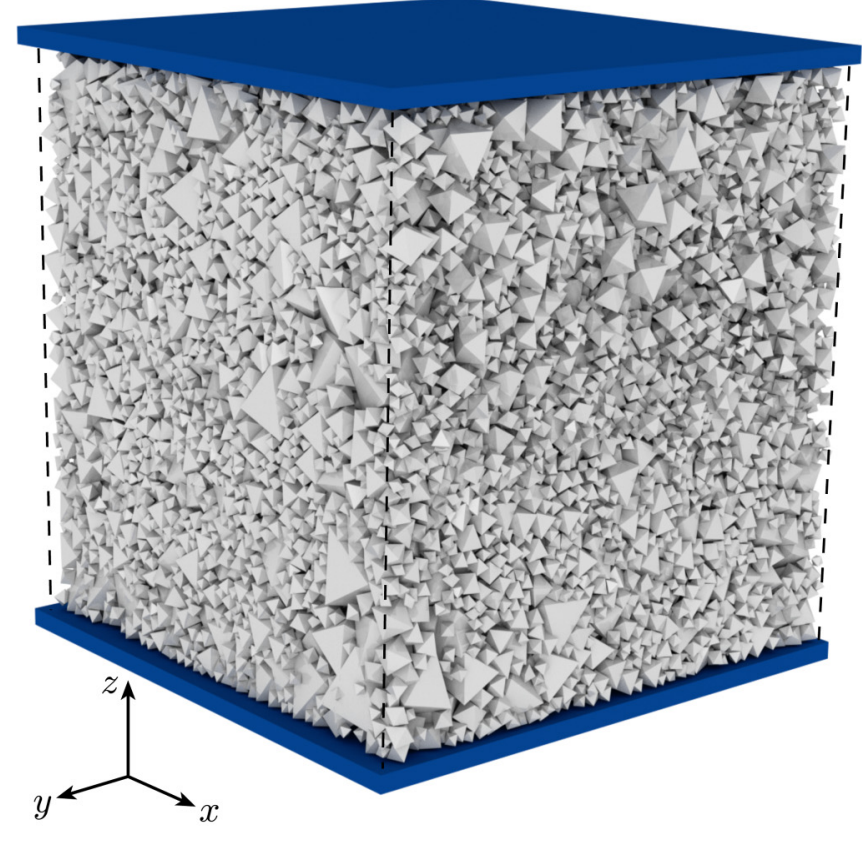

(b)

FIG. 2. Snapshots of samples composed of 19000 octahedra with grain size span corresponding to (a) $S=0.0$ and (b) $S=0.8$.

Though time-consuming, this process improves the quality of the numerical resolution and particle kinematics by adapting the contact common-planes to small adjustments in particle translation and, often more critical, to particle rotations. Nonetheless, the relatively small particle velocities in our tests also allow one to simplify the particle rotation scheme by only considering the linear terms for the body motion updates, as explained in Ref. [45].

The shearing tests continued up to reaching the steady flow state (also known as the critical state in soil mechanics [46]) in which the sample volume and the shear strength only present small variations around well defined mean values. In the case of three-dimensional angular grains, this occurs at relatively high levels of deformation. For this reason, we sheared our samples up to a cumulative shear strain $\gamma \simeq 100 \%$. Although these simulations may take several weeks to run, we also took advantage of the parallel version of the contact dynamics method available in the platform LMGC90 to reduce the computing time. Details of the parallel implementation of the method are available in Ref. [47].

Videos of the shearing tests can be found in the following link: [48].

\section{MACROSCOPIC SHEAR STRENGTH AND SOLID FRACTION}

The steady flow in granular materials is typically characterized by the stabilization of the shear strength and the packing fraction. The shear strength in our tests was determined by using the granular stress tensor

$$
\sigma=\frac{1}{V} \sum_{\forall c} f^{c} \ell^{c},
$$

where $V$ is the volume of the sample and a sum of the dyadic product between the force vector $\boldsymbol{f}$ and the branch vector $\boldsymbol{\ell}$ (i.e., the vector joining the centers of mass of particles in contact) is done over all the contacts $c$. Then, the macroscopic coefficient of friction $\phi^{*}$ was computed on the steady state using the relation

$$
\sin (\phi)^{*}=q / p
$$

with the deviatoric stress $q=\left(\sigma_{1}-\sigma_{2}\right) / 2$, the mean pressure $p=\left(\sigma_{1}+\sigma_{2}\right) / 2$, and $\sigma_{1}$ and $\sigma_{2}$ are the principal stresses on the shear plane $x z$. The upper script $(*)$ indicates parameters obtained as averaged values in the steady flow state. Hence, Eq. (3) is only valid after taking $q$ and $p$ as averaged values in the steady state as well. Note that we assume that stresses are homogeneous along the $y$ axis and we only consider the projections on the shear plane. This is not the case for the transient nonsteady flow (i.e., $\gamma<0.4$ ). In the steady state, the lateral components of the stress tensor may present differences of the order $\sigma_{x x} / \sigma_{y y} \simeq 1.1$. This ratio is small enough to allow us to use, for the sake of simplicity, the characterization of shear strength by only considering the stress components on the shear plane.

Figure 3 shows the evolution of the relative deviatoric stresses $q / p$ as a function of the cumulative shear deformation $\gamma$. For all the samples, we can observe an initial increase of $q / p$ up to a peak strength near $\gamma \sim 0.1$. Then, as the initial packing state of the samples is relatively dense, the samples dilate and the shear strength smoothly decreases and reaches a steady value for $\gamma>0.6$.

The packing fraction $\left(v^{*}=V_{s} / V\right.$ with $V_{s}$ the volume of the grains) smoothly decreases for the nine samples and reaches steady values for deformations $\gamma>0.6$ (see Fig. 4). As expected, the samples presenting a broader variety of grain sizes show a higher density given the capacity of smaller grains to fill the pores between the bigger. Such a difference is not reflected in shear strength, though. Thus, for three- 


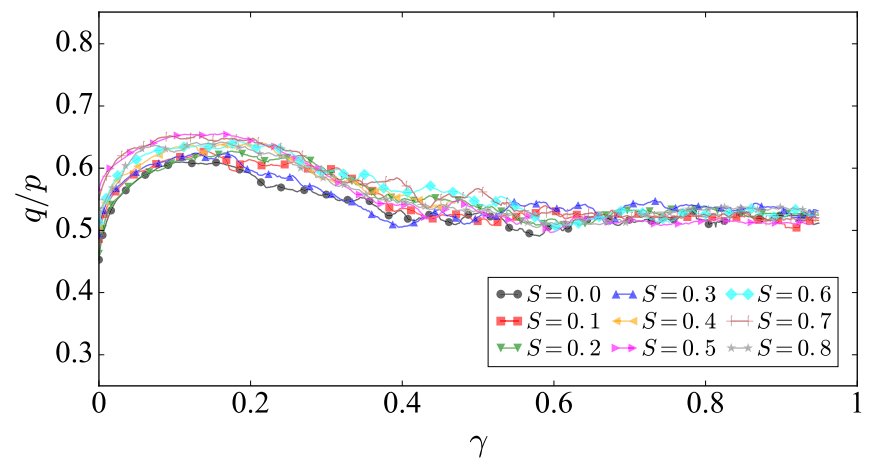

FIG. 3. Evolution of the relative deviatoric stress as a function of the cumulative shear deformation $\gamma$ for the set of samples having different grain size span $S$.

dimensional angular grains, the grain size span does not play either a role on the shear strength of granular assemblies.

Figures 5(a) and 5(b) show the average shear strength and packing fraction as a function of the grain size span $S$ in the steady state for both our results using octahedra and those reported for spheres in Ref. [5]. The average shear strength for octahedra is $\sin (\phi)^{*} \simeq 0.5$ or $\phi^{*} \simeq 30^{\circ}$ compared to $\sin (\phi)^{*} \simeq 0.34$ or $\phi^{*} \simeq 19^{\circ}$ for spheres. In the case of the average solid fraction, the evolution for both particle shapes is nonlinear with the grain size span $S$ showing very similar growing rates except for the highest size spans. Note, however, that from Fig. 4 we can deduce that the dilatancy rates are very similar across grain size spans.

Our results and those reported previously in literature are consistent and confirm the independence of shear strength with grain size span. Still, this phenomenon remains puzzling to observe in physical experiments. It is difficult to control the grain size and shape with real particulate materials or, once the grain size dispersion exceeds a given ratio, standard testing equipment can no longer contain representative samples. Additionally, the grains may suffer abrasion or even fragmentation when sheared. These phenomena may strongly modify the grain shapes and stress transmission resulting in different shear strength. Nonetheless, the understanding of the strength behavior of granular materials relies on the granular structure and contact fabric that we analyze in the following.

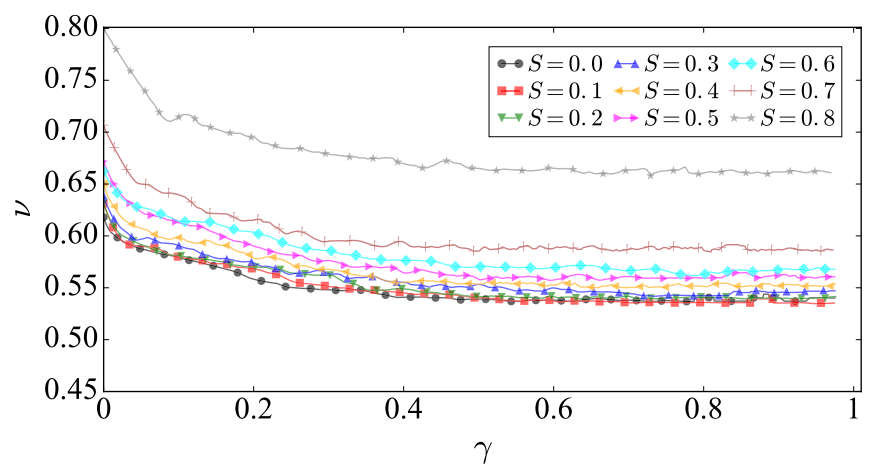

FIG. 4. Evolution of the solid fraction as a function of the cumulative shear strain $\gamma$ for the different grain size spans tested.

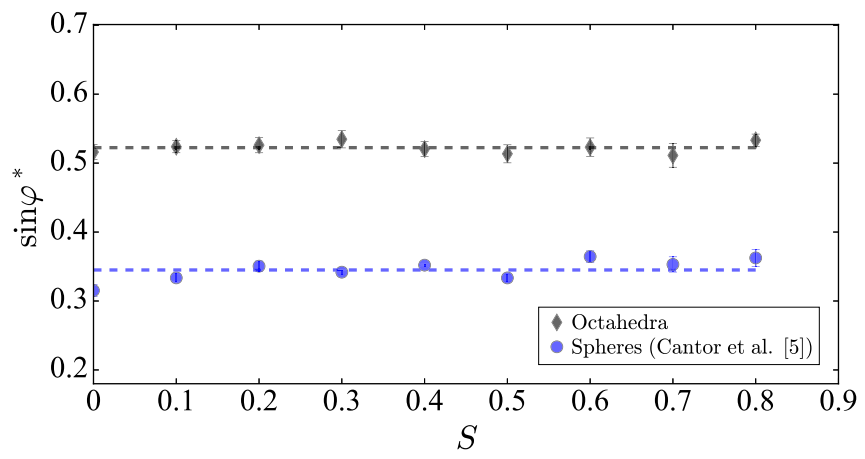

(a)

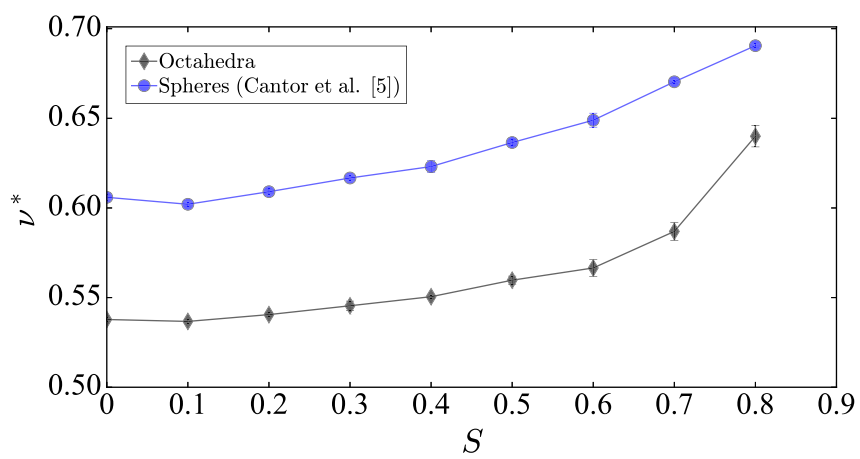

(b)

FIG. 5. (a) Average macroscopic coefficient of friction and (b) average solid fraction as a function of the grain size span at the steady flow state. Error bars represent the standard deviation of the data.

\section{GRANULAR FABRIC}

\section{A. Grain connectivity}

\section{Coordination number}

A first-order characterization of the granular fabric may be done by using the coordination number defined as $Z^{*}=$ $2 N_{c} / N_{p}\left(1-P_{f}\right)$, where $N_{c}$ is the number of force bearing contacts, $N_{p}$ is the number of particles in the sample, and $P_{f}$ is the proportion of floating particles (i.e., grains having less that two force bearing contacts).

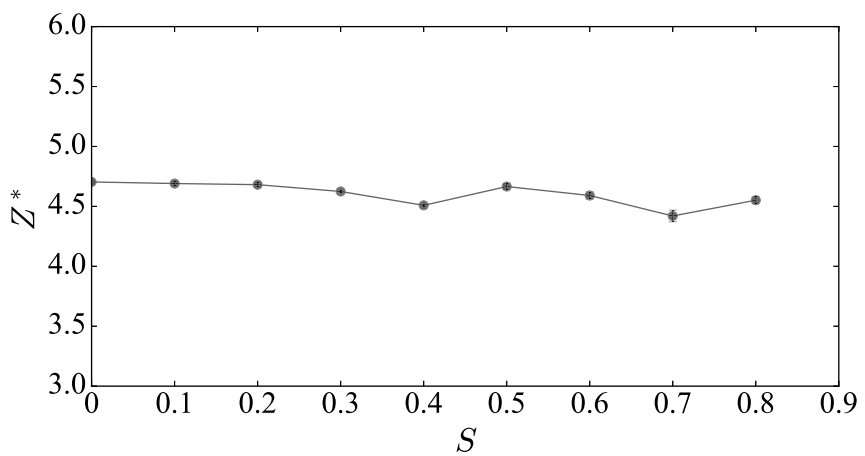

FIG. 6. Average coordination number at the steady state as a function of the grain size span. Error bars, presenting the standard deviation of the date, are practically unnoticeable. 


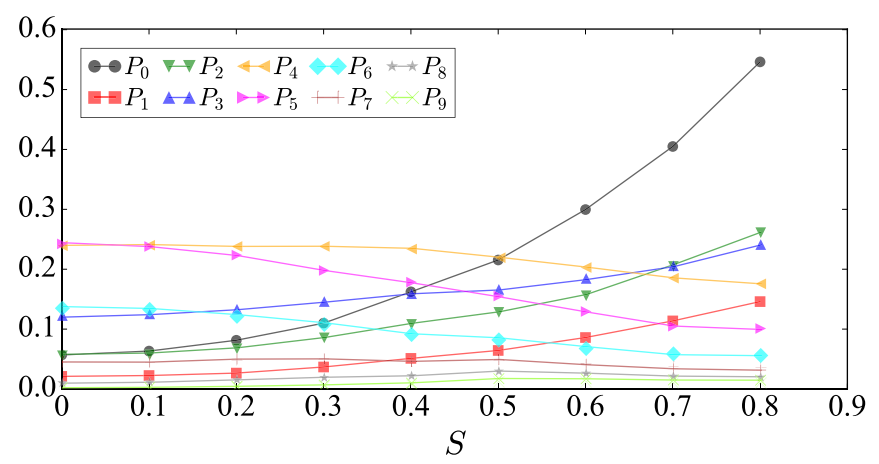

FIG. 7. Average proportion of force bearing contacts $P_{k}$ that particles may support as a function of the grain size span.

Figure 6 presents the average coordination number in the steady state and suggests that for any grain size span the coordination number is invariant under for the inertial number and coefficient of friction considered. This invariance of the coordination number involves complex compensation mechanisms related to the grains' role and size in the contact network. Figure 7 displays the proportion $P_{k}$ of particles having $k$ number of contacts. Although the maximal number of contacts a grain can carry dramatically increases with $S$, we only show the set $k=\{0$ to 9$\}$ for the sake of simplicity. Note, however, that for the sample $S=0.8$, we can find some grains with more than 15 contacts. As also observed in Ref. [49], the proportion of rattlers $P_{f}=P_{0}+P_{1}$ sharply increases with $S$. Indeed, for $S=0.8$, less than half of the particles in the sample are actively participating at the force transmission. Apart from the set of rattlers, the proportion of particles having two or three contacts $\left(P_{2}\right.$ and $\left.P_{3}\right)$ is the set that increases at a higher rate. This set usually corresponds to small-size particles helping to stabilize the granular backbone of the sample. On the other hand, proportions $P_{4}$ to $P_{7}$ decrease with $S$. For $k>7$, the proportions $P_{k}$ slightly increase aside from some particular cases. See, for instance, $P_{8}$ in which its increment reaches a peak and gets stabilized for $S>0.6$.

In fact, we can observe that the macroscopic coordination number, for any grain size span, can be computed by the local contributions of each proportion of grains having a given number of contacts as

$$
Z^{*}=\sum_{k=2}^{N_{c}^{m}} k P_{k},
$$

where the sum is computed over all proportions of contacts up to the maximal number of contacts per grain in the sample $N_{c}^{m}$. Equation (4) is one approach to observe and understand the invariability of coordination across polydisperse samples.

It is possible to observe the correlation size-connectivity in the assembly by computing the average coordination number as a function of the relative size of grains as $d_{r}=(d-$ $\left.d_{\min }\right) /\left(d_{\max }-d_{\min }\right)$. Figure 8 shows the average coordination number by particle sizes $\left(Z_{d_{r}}\right)$ for all the grain size spans. We observe a continuous increase of the particle connectivity occurs as a function of the relative particle size. Big particles are usually surrounded by several small particles more poorly connected but giving important support to the granular backbone. Besides, note that the relative particle

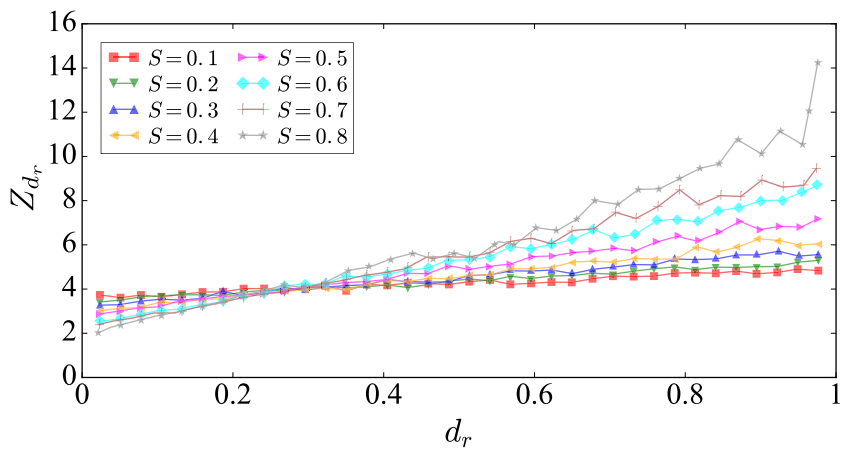

FIG. 8. Average coordination number as a function of the relative particle equivalent diameter in the sample for the different size spans.

size $d_{r} \simeq 0.3$ appears systematically as a typical particle size bearing the average coordination sample through the different samples.

\section{Contributions by contact type}

The independence of the average number of contacts per grain with grain size span is an interesting observation that can be finely analyzed by considering the different types of contacts between grains. As mentioned before, it is possible to find four types of contacts between angular convex grains: vertex-face, edge-edge, edge-face, and face-face. For simplicity, we label these contact types as $v f$, ee, ef, and $f f$, respectively.

Figure 9 shows the contributions to the macroscopic coordination number by contact types so

$$
Z^{*}=Z_{v f}+Z_{e e}+Z_{e f}+Z_{f f} .
$$

This equation reveals that the invariant connectivity is a result of an increment of contacts edge-face and face-face while the single contact type vertex-face and edge-edge decrease. As fewer particles are involved at the stress transmission as $S$ increases (we observed $P_{f}$ rapidly increasing with $S$ ), the force bearing should be principally focusing on contacts $f f$. Contacts $e-e$, occurring in a significant proportion, are mechanically more unstable and seldom seen through the main granular backbone.

It is also interesting to investigate the evolution of particle connectivity by contact type across particle sizes. Figure 10

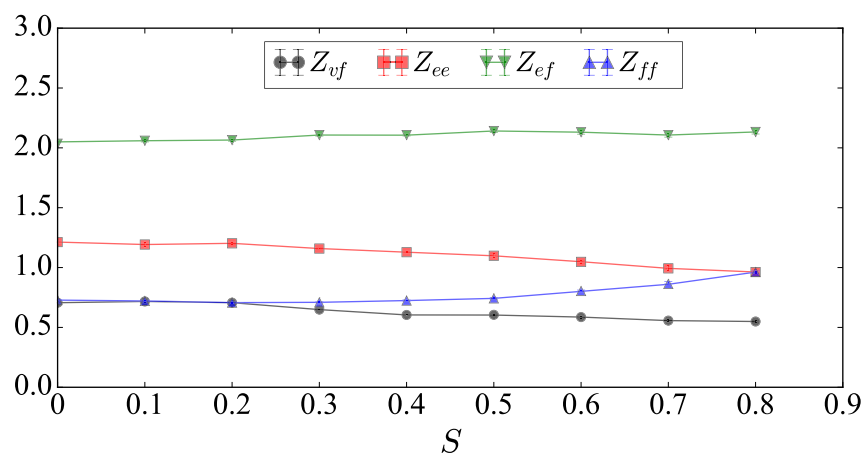

FIG. 9. Coordination decomposition by different contact types and for the different grain size spans tested. 

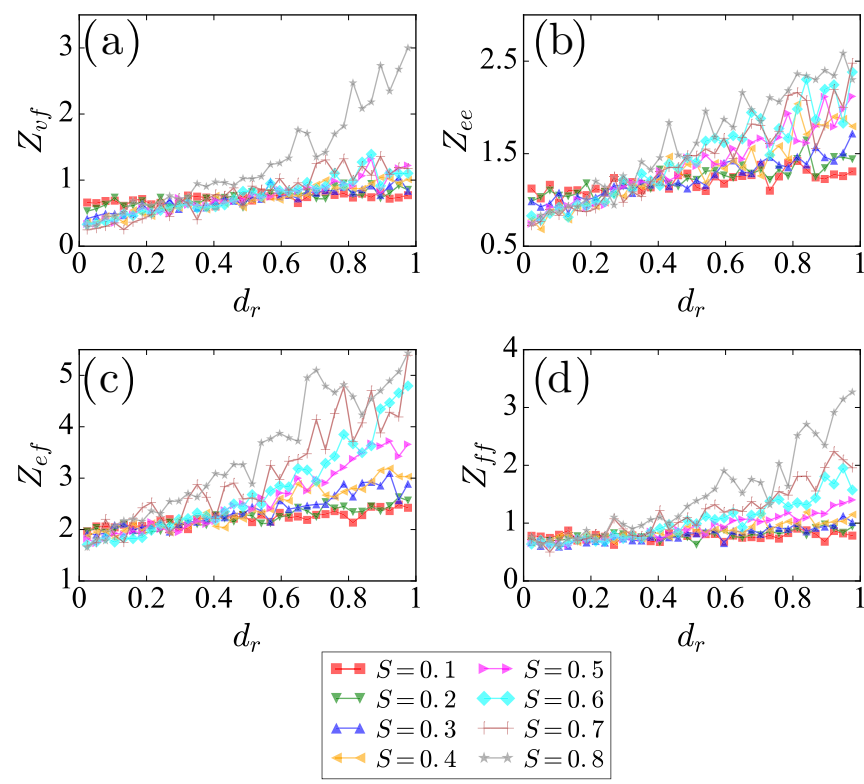

FIG. 10. Evolution of coordination number as a function of the relative particle size $d_{r}$ for contacts (a) vertex-face, (b) edge-edge, (c) edge-face, and (d) face-face.

displays the local coordination number as a function of the relative size $d_{r}$ for the four types of contacts. We observe that for all contact types, a gradual increase of connectivity occurs with particles size. The increase of contacts edgeedge is relatively smoother across particle sizes. Although the slopes of curves for $Z_{e e}$ and $Z_{e f}$ are similar as $S$ increases, the participation in the macroscopic average connectivity of contacts edge-face is more important by number proportion. Note that that type of contact also provides a relatively more stable mechanism for force bearing. In the case of contacts face-face, an important contribution to global connectivity can be observed for the classes of bigger particles. However, for relative particle sizes $d_{r}<0.3$, the connectivity seems to be less linked to the particle size span. Let us highlight that the set of contacts $f f$ is the second most important source of connectivity and, then, of stress transmission in the sample.

For brittle granular materials, the participations to the granular connectivity may dramatically change as single contacts (i.e., edge-face and edge-edge) usually concentrate high stresses, making them prompt to damage and fragmentation. For such cases, the level of stresses applied upon the granular assemblies can play a central role that may allow one, or not, to reproduce the shear strength invariance with grain size span.

\section{B. Contact network}

The variability of the local granular connectivity should be reflected in the contact network. We characterize this network through the microstructural anisotropies linked to the contact orientations and the branch vectors.

\section{Contact anisotropy}

The contact orientations are here described by the probability distribution defined as

$$
P_{c}(\theta)=\frac{N_{c}(\theta)}{N_{c}},
$$

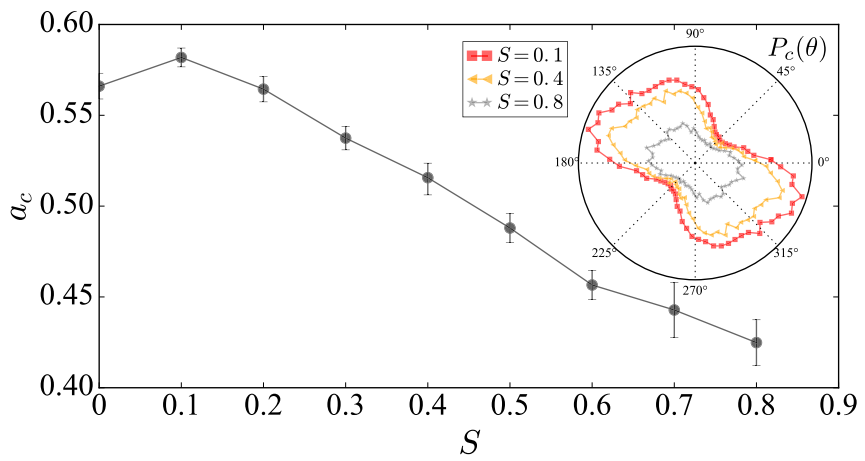

FIG. 11. Evolution of the contact orientation anisotropy as a function of the grain size span. Error bars present the standard deviation of the data. In the inset: polar representation of the probability distribution of contact orientations $P_{c}(\theta)$ for samples $S=0, S=0.4$ and $S=0.8$.

where $N_{c}$ is the number of contacts pointing in the direction $\theta$. Note that we take advantage of the axial symmetry along the $y$ axis. Then, the angle $\theta$ is just the projection of the normal contact vector on the shear plane $x y$. Such angular representation (see inset in Fig. 11), can be characterized with a first-order approximation of the Fourier series as

$$
P(\theta)=\frac{1}{\pi}\left\{1+a_{c} \cos 2\left(\theta-\theta_{c}\right)\right\},
$$

where $\theta_{c} \simeq 135^{\circ}$ is the main orientation of the distribution and $a_{c}$ its anisotropy.

Figure 11 shows the evolution of the contact orientation anisotropy as a function of the grain size dispersion. At first, the anisotropy presents a high value for the monodisperse configuration of grains. Note that the preferential contact orientation and the orientation of its faces may be affecting the $P_{c}$ distribution. Hence, the expected preferred contact orientation $\theta_{c} \pm 30^{\circ}$ may be affected by local arrangements and correlated to the particles' dihedral angles. This observation should not be misunderstood with particle crystallization (ordered arrangement of particles). To avoid such ordering, we added a small grain size dispersion around $S=0$ and the average coordination number, close to that of the polydisperse samples, let us deduce that our system is truly representing a disordered medium.

Back to Fig. 11, we observe that $a_{c}$ gradually drops as $S$ increases. Such a phenomenon is also observed during the compression of polydisperse packings in 2D [50] and 3D [51] simulations. As some grains can be accommodated between the bigger ones, the angular distribution becomes relatively more homogeneous. Its effect can be seen in the inset of Fig. 11 for $S=0.8$, where a more significant proportion of contacts appear in the orthogonal direction of $\theta_{c}$. The previous description of connectivity across particle classes and contact types supports the fact that, locally, bigger grains are surrounded by an increasing number of load-bearing contacts. Then, an important homogenizing effect of contact directions is supported by the presence and concentration of contacts around the largest classes of particles. 


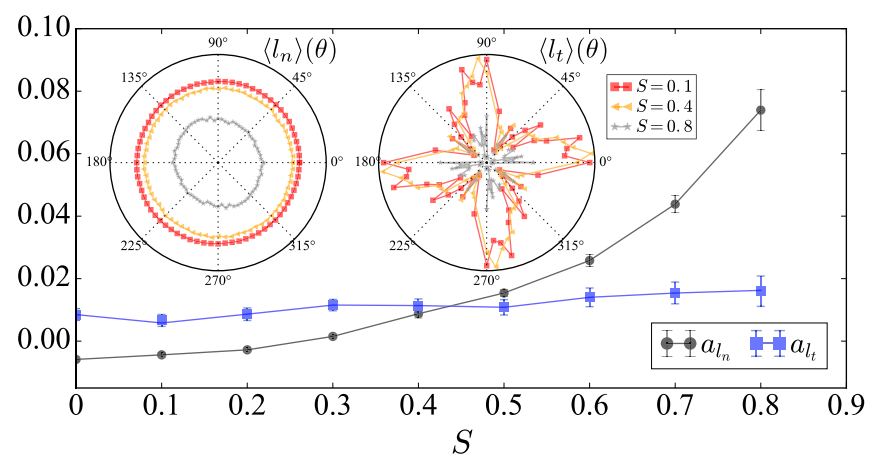

FIG. 12. Evolution of branch anisotropies $a_{l_{n}}$ and $a_{l_{t}}$ as a function of the grain size span. Error bars present the standard deviation of the data. In the inset: polar representation of average branch length projections on the modified contact frame $\boldsymbol{n}^{\prime}$ and $\boldsymbol{t}^{\prime}$.

\section{Branch anisotropies}

The branch network is the structure created by the vectors joining the center of particles having force bearing contacts. Such vectors, in the case of nonspherical particles, have orientations that do not necessarily match those of the normal contact vectors $\boldsymbol{n}$. For coherence with the previous section, the branch vectors $\ell$ are projected on the modified contact local attached to the shear plane $x y$. Let us define the vector $\boldsymbol{n}^{\prime}$ as the projection on the shear plane of the normal contact vector. The corresponding tangential orientation $\boldsymbol{t}^{\prime}$ is defined by a counterclockwise rotation of $90^{\circ}$ of vector $\boldsymbol{n}^{\prime}$ still on the plane $x y$. Thus, the branch vector $\ell$ has "normal" and "tangential" projections defined in the new frame as $\ell_{n}=\boldsymbol{\ell} \cdot \boldsymbol{n}^{\prime}$ and $\ell_{t}=$ $\boldsymbol{\ell} \cdot \boldsymbol{t}^{\prime}$, where (.) is the dot product.

Then, the expressions

$$
\left\langle\ell_{n}\right\rangle(\theta)=\frac{1}{N_{c}(\theta)} \sum_{c \in \delta \theta} \ell_{n}
$$

and

$$
\left\langle\ell_{t}\right\rangle(\theta)=\frac{1}{N_{c}(\theta)} \sum_{c \in \delta \theta} \ell_{t},
$$

describe the probability density functions of branch lengths linked to the normal contact orientation on the shear plane. This computation considers a summation of branch components pointing at the contact orientations $\delta \theta$ (i.e., small intervals of angle in the range $[0,2 \pi]$ ).

The inset of Fig. 12 shows the polar distribution of $\ell_{n}$ and $\ell_{t}$ for three different samples $S=0, S=0.4$ and $S=0.8$. We can observe that the normal branch orientations present a subtle stretching along the orientation $\simeq 135^{\circ}$. The preferential orientations for $\ell_{t}$ are related to the normal branch orientation $\theta_{\ell_{n}} \pm 45^{\circ}$ since tangential branch length is maximal when $\angle(\ell, n)=45^{\circ}$ using octahedra.

Similar to the contact orientations, the branch distributions can be described by expressions

$$
\left\langle\ell_{n}\right\rangle(\theta)=\left\langle\ell_{n}\right\rangle\left\{1+a_{\ell_{n}} \cos 2\left(\theta-\theta_{\ell_{n}}\right)\right\}
$$

and

$$
\left\langle\ell_{t}\right\rangle(\theta)=\left\langle\ell_{n}\right\rangle\left\{a_{\ell_{t}} \sin 2\left(\theta-\theta_{\ell_{t}}\right)\right\}
$$

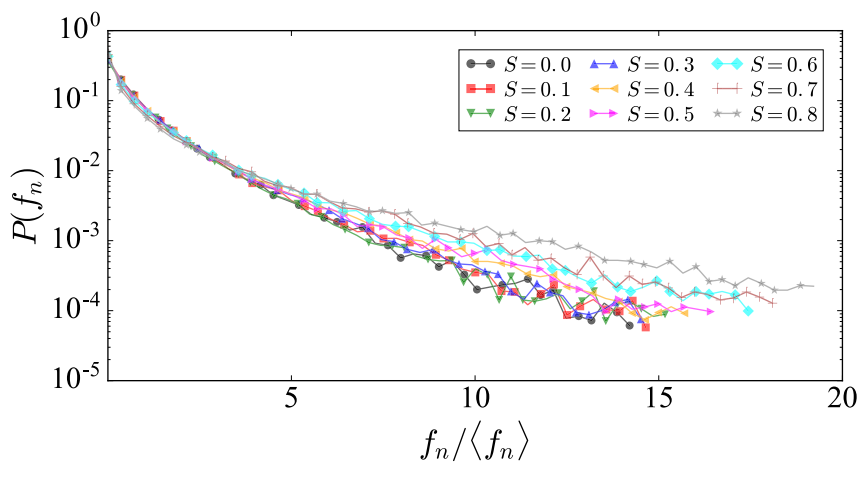

FIG. 13. Probability density functions of normal contact forces normalized by the corresponding mean force for the different grain size spans.

where $a_{\ell_{n}}$ and $a_{\ell_{t}}$ are the normal and tangential branch anisotropies, respectively, and $\theta_{\ell_{n}}$ and $\theta_{\ell_{t}}$ are the principal orientation of each distribution.

Figure 12 presents the evolution of the branch anisotropies as a function of the grain size span. Although both values are relatively small when compared with the contact anisotropy, we observe that $a_{\ell_{n}}$ increases non linearly with $S$ as a consequence of the increasing variability of branch length as particles of different sizes get in contact. Additionally, the tangential branch anisotropy slightly increases with the grain size span and its effect is barely noticeable on the polar representation of $\left\langle\ell_{t}\right\rangle$ (see inset of Fig. 12).

\section{FORCE NETWORK}

The geometrical structure that particles and contacts create is complemented by the contact forces whose properties may be affected by the grain size dispersity.

Consider, for instance, the normal contact force distribution. As observed in Fig. 13, the probability density function of normalized normal forces by the mean force becomes broader as the grain size span increases. During shearing, forces between monodisperse polyhedra can reach up to 15 times the mean normal force while for highly polydisperse samples the maximal normal force could easily exceed 20 times $\left\langle f_{n}\right\rangle$.

Focusing on small forces, Fig. 14 allows us to observe that higher grain size spans produce a more important set of weak forces. Although subtle, such a difference translates the broader force configuration that polydisperse assemblies present. Although a bimodal distribution of forces may be still recognized as typically done in granular media (i.e., an exponential distribution for $\left.f_{n}\right\rangle\left\langle f_{n}\right\rangle$, and a power law for $f_{n}<\left\langle f_{n}\right\rangle$ [52-56]), our study suggests that such transition between regimes dims for samples containing broader grain size distributions. These observations are also consistent with those undertaken on polydisperse packings of spheres under compression in Refs. [57,58]

\section{A. Contributions by contact type}

The contribution to force transmission can be analyzed as well as partitions considering each type of contact. Figure 15 allows us to visualize and highlight the strong heterogeneities 


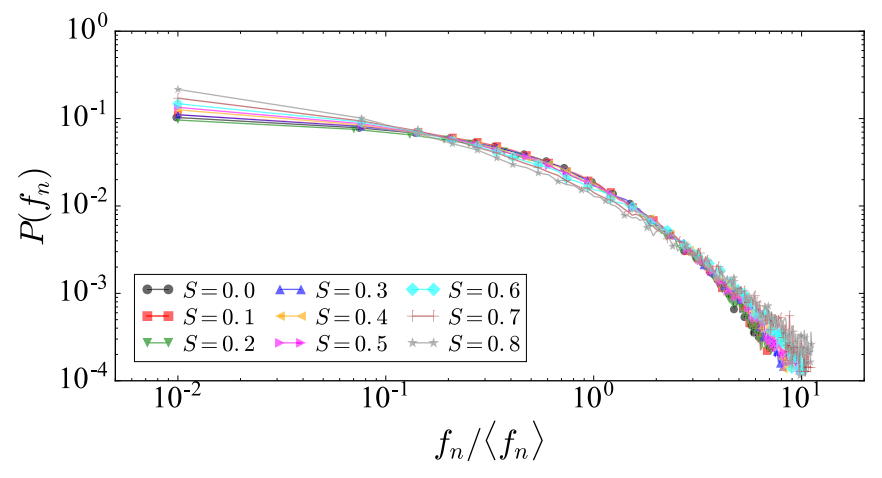

FIG. 14. Probability density functions of normal contact forces in $\log$ - $\log$ scale revealing the evolution of small contact forces for the different grain size spans.

of the force network and the role of contact types in the system.

As mentioned before, the face-face contacts are supporting an important part of the loads, making them easily identifiable (red bars) as they are concentrated along the granular backbone (extended force chains transmitting relatively high proportion of the load between the walls). On the other hand, single point and edge-face contacts are particularly more present in the secondary structure of weak forces (thin bars) bringing support to the backbone.

As a first indicator of the force transmission evolution across grain size spans, Fig. 16 shows the average contact force during the steady state by contact type for each of the size spans. Note that these values are normalized by the average normal contact force in the sample, revealing the relative contributions of each contact type. We can observe that more stable contacts face-face are usually bearing the highest forces above the mean, while contacts vertexface and edge-edge (single point contacts) are supporting, on average, similar levels of forces under the mean. The contacts edge-face bear forces rather close to the mean force.

The evolution of these average contact forces by contact type slightly changes as $S$ increases. Although our discrete approach using rigid grains does not allow us to account for bodies stresses distribution, we can induce that only the contacts type edge-edge may present a relative offload as $\left\langle f_{n}^{e e}\right\rangle$ remains nearly constant and its proportion of contacts relatively increase (see Fig. 9 above). In contrast, the set of contacts for $\left\langle f_{n}^{v f}\right\rangle$ decrease, supporting the fact that these more unstable interactions may present significant stress concentrations.

\section{B. Forces anisotropies}

The variability of force orientations may be captured using the angular distributions of its components. Let us consider the angular variations of normal and tangential force intensities on the shear plane through the expressions

$$
\left\langle f_{n}\right\rangle(\theta)=\frac{1}{N_{c}(\theta)} \sum_{c \in \delta \theta} f_{n}
$$

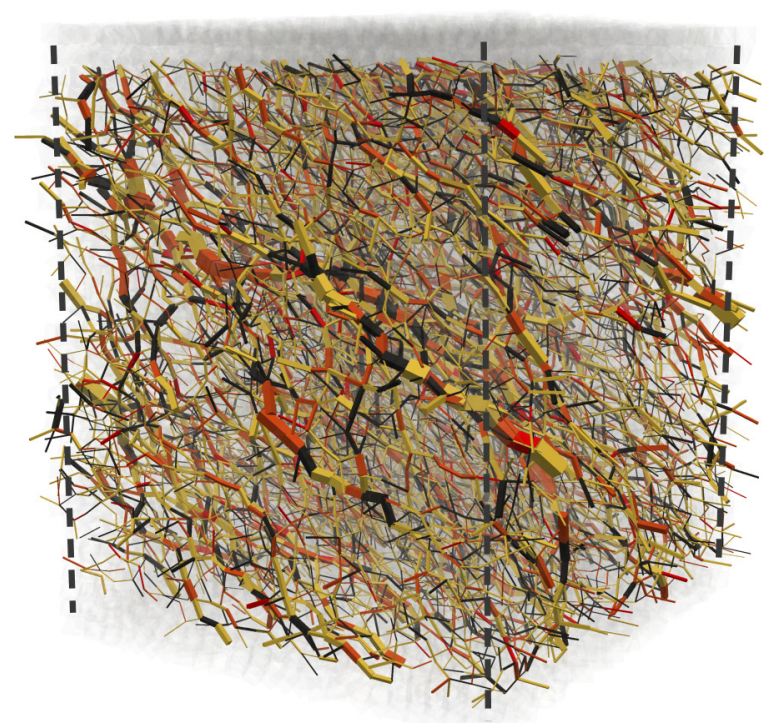

(a)

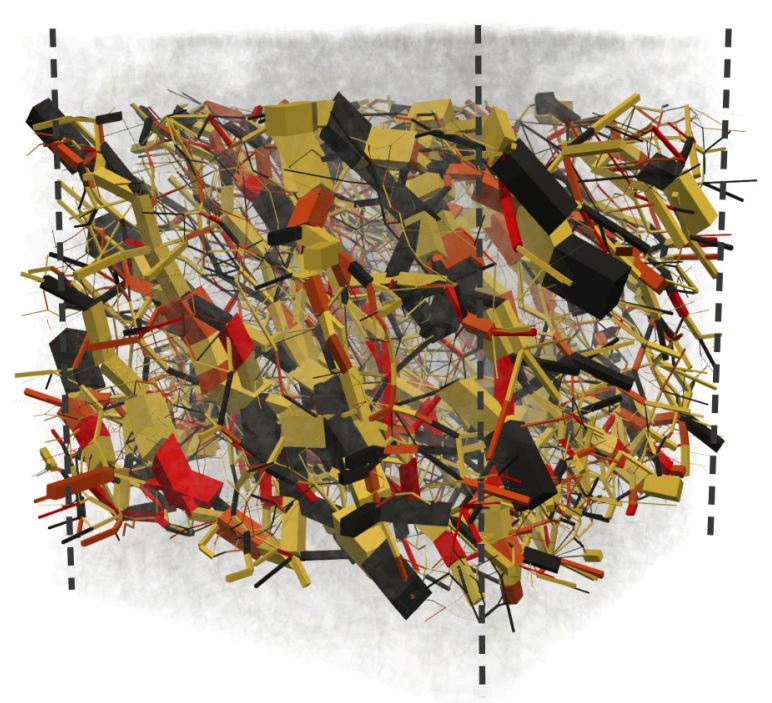

(b)

FIG. 15. Snapshots displaying the distribution of contact types and their role in the force networks for samples (a) $S=0.0$ and (b) $S=0.8$. The contact forces are represented by bars between the centers of grains and their thickness is proportional to the intensity of the normal contact force. Black bars represent vertex-face and edge-edge contacts, yellow bars represent edge-face contacts and red bars represent face-face contacts.

and

$$
\left\langle f_{t}\right\rangle(\theta)=\frac{1}{N_{c}(\theta)} \sum_{c \in \delta \theta} f_{t},
$$

where $f_{n}$ and $f_{t}$ are the intensities of the normal and friction forces. These angular distributions can be observed above of Fig. 17. The intensity of normal contact forces is strongly linked to the contact orientation probability $P_{c}(\theta)$ while the friction forces are preferentially pointing at such orientation $\pm 45^{\circ}$. Akin to the branch distributions, we can approximate 


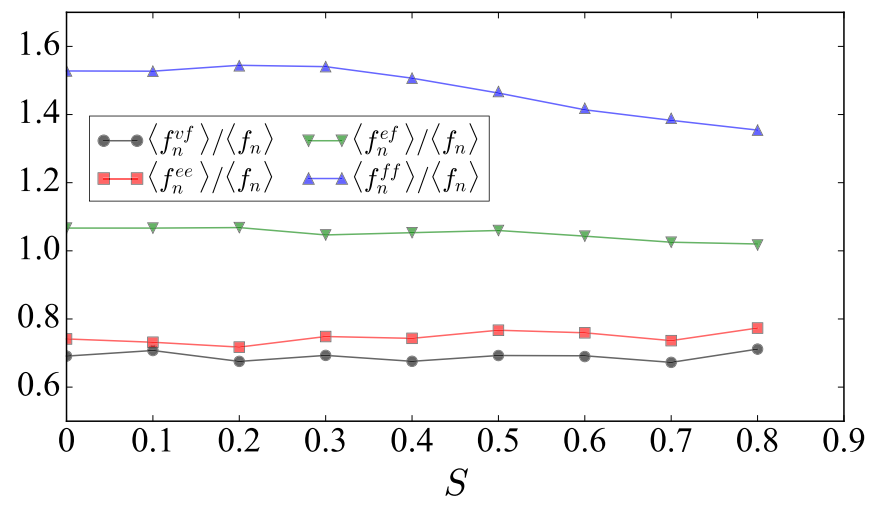

FIG. 16. Average normal contact forces by contact type as a function of the grain size span.

the forces distributions by Fourier series as

$$
\left\langle f_{n}\right\rangle(\theta)=\left\langle f_{n}\right\rangle\left\{1+a_{f_{n}} \cos 2\left(\theta-\theta_{f_{n}}\right)\right\}
$$

and

$$
\left\langle f_{t}\right\rangle(\theta)=\left\langle f_{n}\right\rangle\left\{a_{f_{t}} \sin 2\left(\theta-\theta_{f_{t}}\right)\right\},
$$

where $a_{f_{n}}$ and $a_{f_{t}}$ quantify the anisotropy in each case and $\theta_{f_{n}}$ and $\theta_{f_{t}}$ the preferential orientations.

Figure 17 shows the evolution of the normal and friction forces anisotropies as a function of the grain size span. We observe that the anisotropy of forces increases with the size span and, as observed on the probability density functions of $f_{n}$ (Figs. 13 and 14), it is related to a broadening of contact forces as $S$ increases.

Curiously, the friction forces anisotropy remains rather constant and independent of the grain size span. This is related to the proportion of sliding contacts in the assembly (i.e., contacts where $\left.\left|f_{t}\right| / \mu f_{n}=1\right)$. Such proportion remains constant

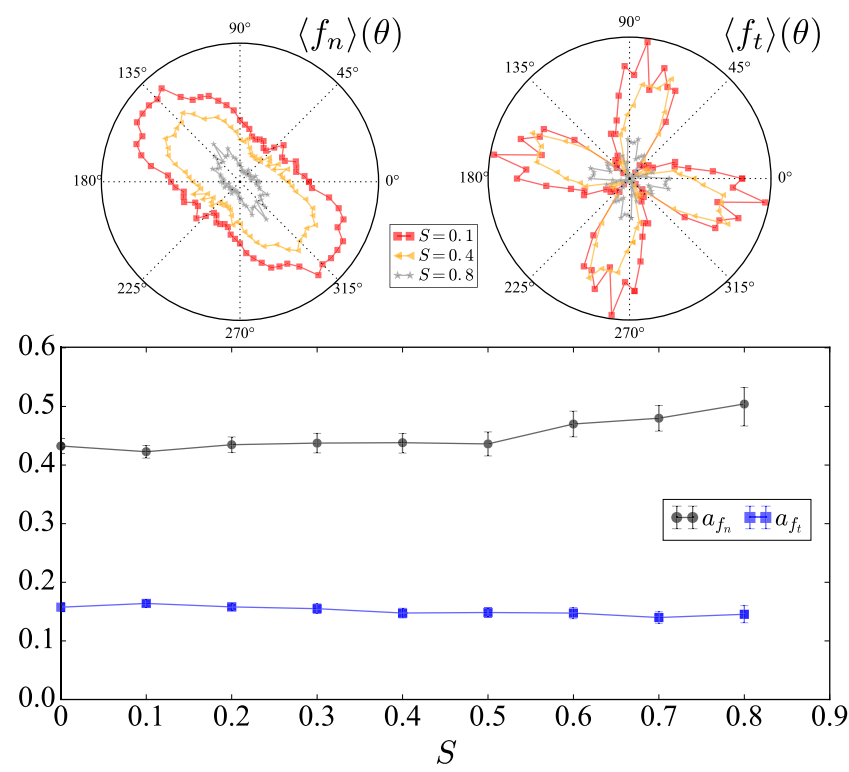

FIG. 17. Evolution of the anisotropy of normal and friction forces as a function of the grain size span. Error bars represent the standard deviation of the data. Above, the polar distribution normal and friction forces for the size spans $S=0, S=0.4$, and $S=0.8$.

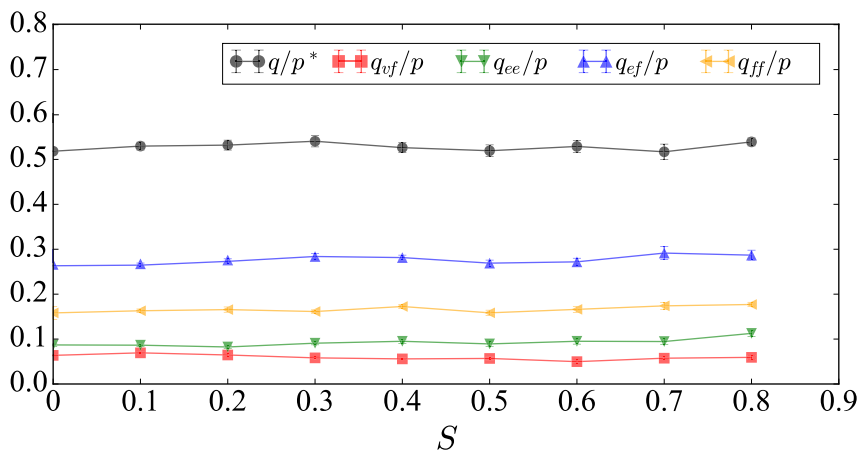

FIG. 18. Contribution of each type of contact to the macroscopic shear strength. The total shear strength (black line) is presented along the contributions of vertex-face contacts (red line), edge-edge contacts (green line), edge-face contacts (blue line), and face-face contacts (yellow line).

through all the spans $S$ and all the contact orientations. This is a fundamental micromechanical similarity that arises from all the samples independently of the size span and is deeply linked to the invariability of the shear strength.

\section{SHEAR STRENGTH DECOMPOSITION}

\section{A. Contributions by contact type}

The macroscopic shear strength can be split to account for the contributions of each contact type as

$$
q / p=\frac{q_{v f}+q_{e e}+q_{e f}+q_{f f}}{p},
$$

where $q_{v f}, q_{e e}, q_{e f}$, and $q_{f f}$ are the deviatoric stresses of partial tensors built after considering only each type of contact. This decomposition lets us estimate the relative levels of stresses carried by families of contacts.

Figure 18 presents the contributions to the deviatoric stresses by contact type in parallel with the total shear strength. We observe that the set of contacts edge-face is supporting the highest level of deviatoric stresses in the assemblies while the set of contacts vertex-face (i.e., more unstable interactions) is supporting relatively lighter stresses. These results are surprising because, despite the complex mechanisms evolving related to the connectivity and the force bearing mechanisms, we can observe that the contributions by family of contacts remain as well independent of the grain size span.

Along with the invariance of the macroscopic coordination number, the stresses partition by contact family is a complementary micromechanical element behind the shear strength independence on the grain size span.

\section{B. Microstructural compensations}

It is well known that a decomposition of the stress tensor [Eq. (2)] is possible employing the angular distribution of branches and contact forces $[1,3,59,60]$. Such analysis yield to the conclusion that the macroscopic shear strength, at the steady flow state, can be estimated through the microstructural 


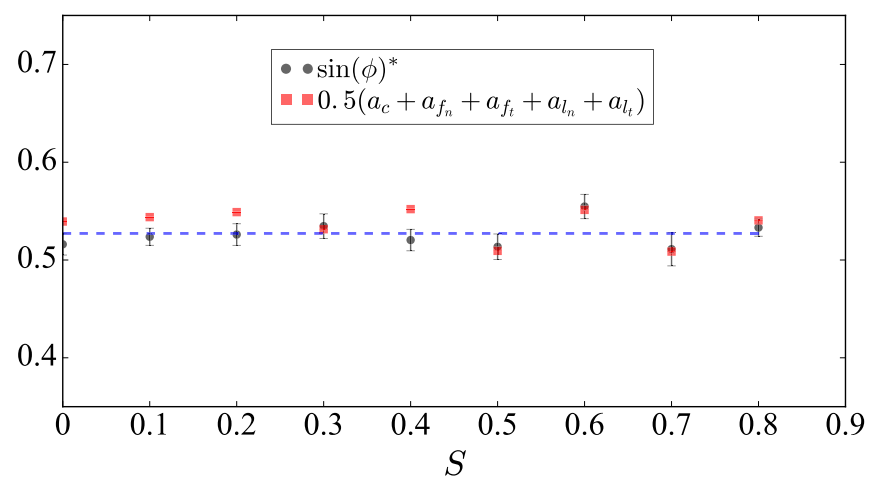

FIG. 19. Macroscopic shear strength $\sin (\phi)^{*}$ and its estimation using the microscopic anisotropies. Error bars represent the standard deviation of the data.

anisotropies as the half sum

$$
\sin (\phi)^{*} \simeq \frac{1}{2}\left(a_{c}+a_{l_{n}}+a_{l_{t}}+a_{f_{n}}+a_{f_{t}}\right) .
$$

Figure 19 presents the macroscopic measures of shear strength using the granular stress tensor and the approximation using the half sum of anisotropies. As it can be observed, this micromechanical decomposition allows us to predict very well the shear strength for our set of samples. Thus, the macroscopic shear strength independence on grain size span can be explained by means of the compensations between the drop of contact anisotropy, the increase of normal forces anisotropy, and a subtle increment in the branch anisotropies. Outstandingly, geometrical and force bearing elements are meeting at the grain and contact scale to produce such invariant macroscopic behavior. The shear strength decomposition reveals the importance of both the fine characterization of granular fabric and the suitability of the contacts dynamic approach to simulate and analyze geometrical and micromechanical properties linked to the particle kinematics and contacts network.

Note, however, that Eq. (17) contains some hypotheses and simplifications that are important to mention. On the one hand, instead of working with the spherical harmonics of the 3D distribution of contacts, branches, and forces orientations, we are simplifying our analysis by projecting these vectors on the shear plane. This allows us to use simpler Fourier series for angular distributions. In a generalized 3D case, the branch vector may present quite different orientation respect to the corresponding force vector. In effect, an orthonormal component to the $\boldsymbol{n}-\boldsymbol{t}$ plane may exist and a third branch orientation (and anisotropy) should be considered. In our simulations, we observed that such a third component of $\ell$ is minimal and may be discarded of the analysis. For more complex samples considering nonconvex or platy grains, Eq. (17) may no longer hold up in part because of disregarding that additional branch anisotropy.

Also, we assume as well that the fabric tensor and stress tensor present equal principal orientations. This is not necessarily true during the transient nonsteady flow. For this reason, this analysis is strictly restrained to the steady state. Finally, during the development of the stress tensor decomposition, some higher-order products are ignored for the sake of simplicity of the micro-macro relation. See Ref. [10] for a detailed accounting of high order microstructural anisotropies.

\section{SUMMARY AND PERSPECTIVES}

We developed a set of numerical experiments in the framework of the discrete element approach known as contact dynamics. We built a set of nine samples composed of angular rigid bodies (octahedra), where the grain size span varied from a configuration containing equal size particles up to one containing a uniform distribution of sizes and a ratio maximum/minimum equivalent diameter $\sim 10$.

By means of periodic shearing of samples composed of 19000 grains, we analyzed the steady flow state under quasistatic conditions. It allowed us to generalize to threedimensional angular grains the observation that grain size span does not affect the shear strength although the solid fraction increases as a broader grain size distribution is considered.

To explore the microstructural elements behind such behavior, we started by analyzing the grain connectivity in terms of coordination number, the proportions of grains bearing a given number of contacts, and the proportion of contacts vertex-face, edge-edge, edge-face, and face-face. Such analysis revealed that the mean number of contacts per grain also remains invariable with the size span. This stability of the coordination number is explained by increments of lowcoordinated particles having two or three contacts and a drop in more highly coordinated particles bearing four contacts or more. A family of very connected particles, bearing more than nine contacts, is also created as the grain size span increases. However, their proportion is less significant in the description of the invariability of the macroscopic mean coordination number with respect to the low-coordinated grains.

We also described the intergranular force bearing in terms of probability distribution functions of normal contact forces. Such analysis revealed a broadening of the normal contact intensities as the grain size span is increased both for very large and very small forces.

The contact and forces networks were as well analyzed considering their orientations in space. By means of an analysis on the shear plane, we could measure the anisotropies related to the contact orientations, the branch lengths, and the normal and friction forces. In particular, as the grain size span increases, the contact anisotropy drops as a consequence of a richer contact configuration (small grains getting in the poral space created by bigger grains) while the normal contact anisotropy increases (broadening of the contact force range), together with the normal and tangential branch anisotropies. Finally, we used a decomposition of the stress tensor to explain the macroscopic shear strength in terms of structural elements (the anisotropies). Such analysis indicated that the compensations between the geometrical anisotropies (contact and branch) and the force transmission anisotropies nicely explain the invariance of shear strength with grain size dispersion.

Throughout this article, we insisted on the force bearing characteristics of each contact type regarding their connectivity and the relative levels of stress that they can support. The modeling used in this paper does not allow to account 
explicitly for stress distribution inside the bodies. However, the modeling and analysis of the effect of size and shape polydispersity with grains that may break is an interesting perspective that will conduct to a conciliation between numerical approaches, experimental setups and observations.

\section{ACKNOWLEDGMENTS}

This work was supported by a postdoctoral fellowship at Chiang Mai University, Thailand. Also, we counted with the support of the High-Performance Computing Platform MESO@LR.
[1] C. Voivret, F. Radjai, J. Y. Delenne, and M. S. El Youssoufi, Phys. Rev. Lett. 102, 178001 (2009).

[2] N. Estrada, Phys. Rev. E 94, 062903 (2016).

[3] E. Azéma, S. Linero, N. Estrada, and A. Lizcano, Phys. Rev. E 96, 022902 (2017).

[4] D. H. Nguyen, E. Azéma, P. Sornay, and F. Radjai, Phys. Rev. E 91, 032203 (2015).

[5] D. Cantor, E. Azéma, P. Sornay, and F. Radjai, Phys. Rev. E 98, 052910 (2018).

[6] S. Torquato and Y. Jiao, Phys. Rev. E 80, 041104 (2009).

[7] J. Baker and A. Kudrolli, Phys. Rev. E 82, 061304 (2010).

[8] N. Estrada, E. Azéma, F. Radjai, and A. Taboada, Phys. Rev. E 84, 011306 (2011).

[9] E. Azéma, N. Estrada, and F. Radjai, Phys. Rev. E 86, 041301 (2012).

[10] E. Azéma, F. Radjai, and F. Dubois, Phys. Rev. E 87, 062203 (2013).

[11] S. Zhao, X. Zhou, and W. Liu, Granular Matter 17, 793 (2015).

[12] S. Zhao, T. M. Evans, and X. Zhou, Int. J. Solids Struct. 150, 268 (2018).

[13] Z. Lu, A. Yao, A. Su, X. Ren, Q. Liu, and S. Dong, Eng. Geol. 253, 36 (2019).

[14] R. K. Kandasami and T. G. Murthy, Granular Matter 19, 21 (2017).

[15] G. Guida, D. Sebastiani, F. Casini, and S. Miliziano, Géotechnique Lett. 9, 245 (2019).

[16] K. A. Murphy, K. A. Dahmen, and H. M. Jaeger, Phys. Rev. X 9, 011014 (2019).

[17] K. M. Salerno, D. S. Bolintineanu, G. S. Grest, J. B. Lechman, S. J. Plimpton, I. Srivastava, and L. E. Silbert, Phys. Rev. E 98, 050901(R) (2018).

[18] Y. Yuan, L. Liu, Y. Zhuang, W. Jin, and S. Li, Phys. Rev. E 98, 042903 (2018).

[19] A. Sezer, S. Altun, and B. A. Göktepe, Soils Found. 51, 857 (2011).

[20] T. T. Ng, W. Zhou, G. Ma, and X. L. Chang, Int. J. Solids Struct. 135, 74 (2018).

[21] Y. Li, Bull. Eng. Geol. Environ. 72, 371 (2013).

[22] M. D. Jiang, Z. X. Yang, D. Barreto, and Y. H. Xie, Granular Matter 20, 80 (2018).

[23] S. Linero Molina, E. Azéma, N. Estrada, S. Fityus, J. Simmons, and A. Lizcano, Géotechnique Lett. 9, 1 (2019).

[24] J. Gong, J. Liu, and L. Cui, Powder Technol. 353, 178 (2019).

[25] M. Jean and J. J. Moreau, in Unilateral Problems in Structural Analysis-2, International Centre for Mechanical Sciences, Vol. 304, edited by G. Del Piero and F. Maceri (Springer, Vienna, 1987), pp. 151-196.

[26] M. Jean, Comput. Methods Appl. Mech. Eng. 177, 235 (1999).
[27] F. Radjai and V. Richefeu, Mech. Mater. 41, 715 (2009).

[28] F. Radjai and F. Dubois, Discrete-element Modeling of Granular Materials (ISTE Ltd and John Wiley \& Sons Inc., New York, 2011).

[29] F. Dubois, V. Acary, and M. Jean, Comptes Rendus Mecanique 346, 247 (2018).

[30] C. Nouguier, C. Bohatier, J. J. Moreau, and F. Radjai, Granular Matter 2, 171 (2000).

[31] I. Bratberg, F. Radjai, and A. Hansen, Phys. Rev. E 66, 031303 (2002).

[32] S. McNamara and H. Herrmann, Phys. Rev. E 70, 061303 (2004).

[33] B. Chetouane, F. Dubois, M. Vinches, and C. Bohatier, Int. J. Numer. Methods Eng. 64, 65 (2005).

[34] A. Taboada, K.-J. Chang, F. Radjaï, and F. Bouchette, J. Geophys. Res. Solid Earth 110, B09202 (2005).

[35] G. Saussine, C. Cholet, P. Gautier, F. Dubois, C. Bohatier, and J. Moreau, Comput. Methods Appl. Mech. Eng. 195, 2841 (2006).

[36] A. Ries, D. E. Wolf, and T. Unger, Phys. Rev. E 76, 051301 (2007).

[37] V. Topin, F. Dubois, Y. Monerie, F. Perales, and A. Wachs, J. Non-Newton. Fluid 166, 63 (2011).

[38] R. I. Leine, A. Schweizer, M. Christen, J. Glover, P. Bartelt, and W. Gerber, Multibody Syst. Dyn. 32, 241 (2014).

[39] P. A. Cundall, Int. J. Rock Mech. Min. Sci. 25, 107 (1988).

[40] F. Dubois et al., LMGC90 page, https://git-xen.lmgc.univmontp2.fr/lmgc90/lmgc90_user/wikis/home (2020) [Accessed 23-Mar-2020].

[41] F. Dubois, M. Jean, M. Renouf, R. Mozul, A. Martin, and M. Bagnéris, in 10e colloque national en calcul des structures (Computational Structural Mechanics Association, 2011), p. 8.

[42] C. Voivret, F. Radjai, J. Y. Delenne, and M. S. El Youssoufi, Phys. Rev. E 76, 021301 (2007).

[43] K. C. Smith, T. S. Fisher, and M. Alam, Phys. Rev. E 84, 030301(R) (2011).

[44] K. C. Smith, I. Srivastava, T. S. Fisher, and M. Alam, Phys. Rev. E 89, 042203 (2014).

[45] R. Hart, P. A. Cundall, and J. Lemos, Int. J. Rock Mech. Min. 25, 117 (1988).

[46] D. Wood, Soil Behaviour and Critical State Soil Mechanics (Cambridge University Press, Cambridge, 1990).

[47] M. Renouf, F. Dubois, and P. Alart, J. Comput. Appl. Math. 168, 375 (2004).

[48] https://youtu.be/JRAIqueC2sQ.

[49] N. Kumar, O. I. Imole, V. Magnanimo, and S. Luding, Particuology 12, 64 (2014).

[50] N. Estrada and W. F. Oquendo, Phys. Rev. E 96, 042907 (2017).

[51] J. Wiacek and M. Stasiak, Granular Matter 20, 17 (2018). 
[52] D. M. Mueth, H. M. Jaeger, and S. R. Nagel, Phys. Rev. E 57, 3164 (1998).

[53] G. Løvoll, K. J. Måløy, and E. G. Flekkøy, Phys. Rev. E 60, 5872 (1999).

[54] S. G. Bardenhagen, J. U. Brackbill, and D. L. Sulsky, Phys. Rev. E 62, 3882 (2000).

[55] L. E. Silbert, G. S. Grest, and J. W. Landry, Phys. Rev. E 66, 061303 (2002).
[56] L. E. Silbert, Phys. Rev. E 74, 051303 (2006).

[57] R. K. Desu and R. K. Annabattula, Granular Matter 21, 29 (2019).

[58] J. Wiacek and M. Molenda, Int. J. Solids Struct. 51, 4189 (2014).

[59] L. Rothenburg and R. J. Bathurst, Géotechnique 39, 601 (1989).

[60] H. Ouadfel and L. Rothenburg, Mech. Materials 33, 201 (2001). 